\title{
B7 homolog 3 aggravates brain injury in a murine model of Streptococcus pneumoniae-induced meningitis
}

\author{
XUQIN CHEN $^{1,2}$, YANPING WANG $^{1}$, ZHEDONG WANG $^{1}$, RUHONG YAN $^{3}$, JIE LIU $^{1}$, \\ XIANGYING MENG ${ }^{1}$, YAN LI ${ }^{1}$, JIANGHUAI WANG ${ }^{4}$ and JIAN WANG ${ }^{4}$ \\ ${ }^{1}$ Department of Neurology, Children's Hospital of Soochow University, Suzhou, Jiangsu 215003; \\ ${ }^{2}$ Jiangsu Key Laboratory of Translational Research and Therapy for Neuro-Psycho-Diseases, \\ Institute of Neuroscience, Soochow University, Suzhou, Jiangsu 215006; ${ }^{3}$ Department of Clinical Laboratory, \\ The Second Affiliated Hospital of Soochow University, Suzhou, Jiangsu 215004; ${ }^{4}$ Institute of Pediatric Medicine, \\ Children's Hospital of Soochow University, Suzhou, Jiangsu 215003, P.R. China
}

Received August 8, 2014; Accepted February 13, 2015

DOI: $10.3892 /$ etm.2015.2333

\begin{abstract}
Despite the application of antibiotics, Streptococcus pneumoniae (SP)-induced meningitis continues to be a life-threatening disease with a high fatality rate and an elevated risk of serious neurological sequelae, particularly in developing countries. In this study, the contribution of the co-stimulatory molecule B7 homolog 3 (B7-H3) to the pathogenesis of experimental SP-induced meningitis was investigated. Mice were challenged with the intracerebroventricular injection of serotype $3 \mathrm{SP}$ with or without B7-H3. The clinical status of mice with SP-induced meningitis was examined by body weight loss and spontaneous motor activity with neurological scoring. Coronal brain sections were analyzed by counting Nissl-positive neurons and terminal deoxynucleotidyl transferase-mediated dUTP nick end-labeling (TUNEL)-positive cells. Protein expression of neuron-specific enolase (NSE) and $\mathrm{S} 100 \mathrm{~B}$ in brain tissues was examined with immunohistochemical staining. All experiments were performed in a randomized and blinded setting. By the intracerebroventricular injection of SP suspension, a murine model of pneumococcal meningitis was successfully established. In this SP-induced meningitis model, B7-H3 deteriorated the clinical status, as manifested by a decreased neurological score and increased body weight loss. Following the B7-H3 challenge, the number of Nissl-positive cells decreased and TUNEL-stained positive cells increased in the brain tissues of mice with SP meningitis, which demonstrates the enhancement of neuronal necrosis and apoptosis, respectively. Protein expression of NSE was decreased, while that of S100B was increased. These in vivo findings indicate
\end{abstract}

Correspondence to: Dr Jian Wang, Institute of Pediatric Medicine, Children's Hospital of Soochow University, 303 Jingde Road, Suzhou, Jiangsu 215003, P.R. China

E-mail:wj196312@vip.163.com

Key words: B7-homolog 3, Streptococcus pneumoniae meningitis, neuron-specific enolase, S100B, brain injury that B7-H3 aggravates brain injury during the pathological process of experimental SP-induced meningitis.

\section{Introduction}

Despite treatment with effective antibiotics, Streptococcus pneumoniae (SP) meningitis remains a serious infectious disease of the central nervous system (CNS) with mortality rates between 16 and $37 \%$ (1) and neurological sequelae in up to $30 \%$ of survivors (2). Therefore, further study of the pathological mechanisms of SP meningitis and the identification of new means of intervention has a great clinical significance. SP-induced meningitis can cause disruption of the blood-brain barrier (BBB), and most severely it can result in brain damage, which affects the prognosis of patients with SP meningitis. Emerging studies have demonstrated that necrosis and apoptosis of brain neurons occur while brain damage is taking place. Neuron-specific enolase (NSE) and S100B have been confirmed to be specific markers of brain damage (3). B7 homolog 3 (B7-H3) is a newly identified member of the B7 superfamily, which serves a key function in the regulation of immune responses (4). Our previous study (5) found an abnormally high expression level of soluble B7-H3 (sB7H3) protein in children with bacterial meningitis. In a murine model of SP-induced meningitis, we also demonstrated that B7-H3 further augmented the inflammatory response, exacerbated $\mathrm{BBB}$ disruption, and aggravated the pathological injury associated with the disease (6). In order to investigate the impact of $\mathrm{B} 7-\mathrm{H} 3$ on brain damage during SP-induced meningitis, the present study examined the effect of B7-H3 on neuronal necrosis and apoptosis and the protein expression of NSE and S100B in brain tissues in a murine model of SP-induced meningitis.

\section{Materials and methods}

SP-induced meningitis in mice. Pyrogen-free, 8-10-week-old $\mathrm{BALB} / \mathrm{c}$ mice were purchased from SLAC (Shanghai, China). The mice were housed in barrier cages under controlled environmental conditions (12/12 h light/dark cycle, $55 \pm 5 \%$ humidity, $23^{\circ} \mathrm{C}$ ) in the Institute of Medical Biotechnology, 

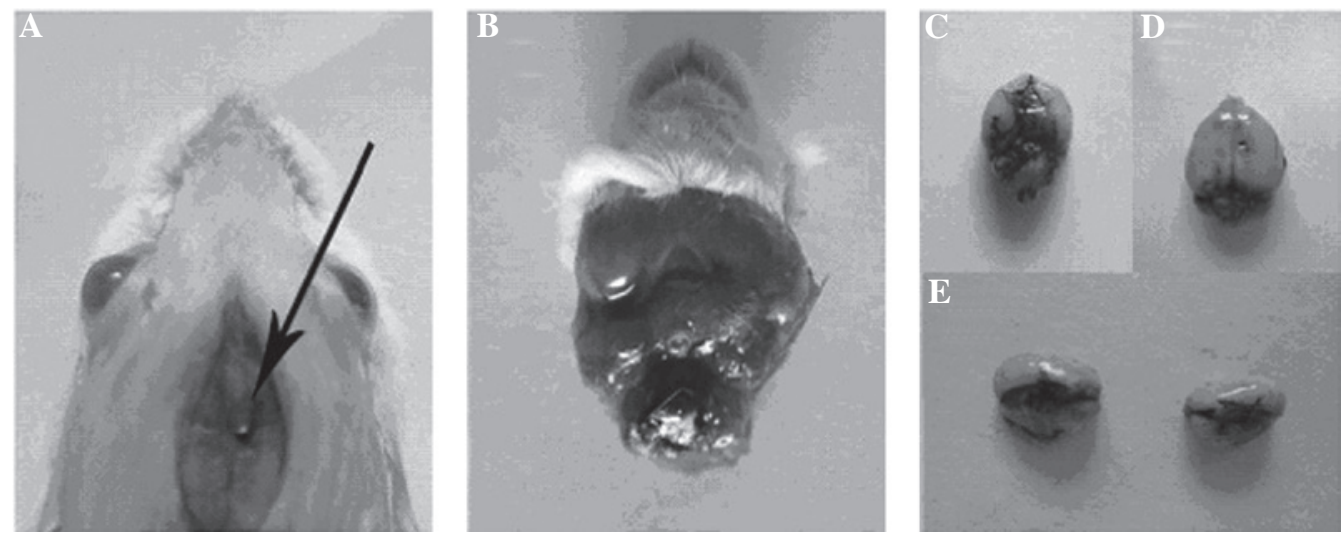

Figure 1. Mouse model of pneumococcal meningitis. (A) Live Streptococcus pneumoniae, or live S. pneumoniae plus B7-H3 was administered via intracerebroventricular injection as described in materials and methods. Injected fluid (colored with black ink) distributes throughout the CSF including (B) intrathecally, (C) in basal spaces, (D) in the perimesencephalic area and (E) in the lateral ventricle. B7-H3, B7 homolog 3; CSF, cerebrospinal fluid.

Soochow University (Suzhou, China) and had free access to standard laboratory chow and water. All animal procedures were carried out in the Institute of Medical Biotechnology, Soochow University. The institutional animal care and use committee of Soochow University Academic Medical Center approved all experiments and all animal studies were conducted with ethical approval granted by the Soochow University Ethics Committee. In addition, the study was conducted in accordance with the recommendations of the Guide for the Care and Use of Laboratory Animals of the National Institutes of Health. Ageand weight-matched BALB/c mice were anesthetized by the intraperitoneal injection of $0.4 \mathrm{ml} 1.8 \%$ Avertin. Pneumococcal meningitis was induced by the intracerebroventricular injection of $15 \mu 1$ sterile normal saline (NS) containing $0.5 \times 10^{4} \mathrm{CFU} / \mathrm{ml}$ serotype 3 SP (American Type Culture Collection, Manassas, VA, USA) into the lateral ventricle as described previously (2). Black ink (\#440; Shanghai Fine Stationery, Co., Ltd., Shanghai, China) injection at the base of the skull was used to confirm that injected fluids were distributed throughout the cerebrospinal fluid.

Grouping and evaluation of clinical disease status. Mice were randomized into one of the following four experimental groups, and each mouse received an intracerebroventricular injection of $15 \mu \mathrm{l}$ in total: i) Control group, injected with $15 \mu \mathrm{l}$ normal saline (NS); ii) B7-H3 group, injected with $15 \mu 1 \mathrm{NS}$ containing $3.3 \mu \mathrm{g}$ recombinant mouse B7-H3 (R\&D Systems, Minneapolis, MN, USA); iii) SP group, injected with $15 \mu \mathrm{lNS}$ containing $0.5 \times 10^{4} \mathrm{CFU} / \mathrm{ml} \mathrm{SP}$; and iv) SP plus B7-H3 group, injected with $5 \mu 1 \mathrm{NS}$ containing $1.5 \times 10^{4} \mathrm{CFU} / \mathrm{ml} \mathrm{SP}$ and $10 \mu 1 \mathrm{NS}$ containing $3.3 \mu \mathrm{g} \mathrm{B7-H3}$. Mice were weighed, put into cages, allowed to wake up, and evaluated clinically at 18 , 48 and $72 \mathrm{~h}$ post SP infection. The clinical disease status was examined by body weight loss and spontaneous motor activity. The following scores were used to assess spontaneous motor activity of mice as described previously (7): 5 points, normal motor activity and turned upright in $<5 \mathrm{sec}$ when put on its back; 4 points, decreased spontaneous activity, but turned upright in $<5 \mathrm{sec} ; 3$ points, turned upright in $>5 \mathrm{sec}$; 2 points, did not turn upright; and 1 point, did not move or coma. At 18 , 48 and $72 \mathrm{~h}$ post SP infection, mice were sacrificed by cervical dislocation. The brain of each animal was removed, and half of the brain was fixed immediately with $4 \%$ formalin for histopathological analysis and immunohistochemical staining.

Histopathology. Brain samples fixed with $4 \%$ formalin were routinely processed, and embedded in paraffin. Sections $(4 \mu \mathrm{m})$ were cut using a microtome (Leica Biosystems $\mathrm{GmbH}$, Wetzlar, Germany), and neuronal loss and cell apoptosis in the cortex were evaluated by Nissl and terminal deoxynucleotidyl transferase-mediated dUTP nick end-labeling (TUNEL) staining (8). Normal neurons were identified by the presence of Nissl bodies in the cytoplasm, loose chromatin and prominent nucleoli. Injured neurons were identified by the loss of Nissl bodies and cavitation around the nucleus. TUNEL staining using an in situ apoptosis detection kit was performed to investigate the apoptosis according to the manufacturer's instructions (Merck, Darmstadt, Germany).

Immunohistochemical analysis. Brain tissue sections ( $4 \mu \mathrm{m})$ were deparaffinized and rehydrated with graded ethanol. For immunohistochemical staining of NSE and S100B, goat anti-NSE (ab90473) and anti-S100B (ab41458) polyclonal antibodies (Abcam, Cambridge, MA, USA) were used at a dilution of 1:100, according to the manufacturer's instructions. Sections incubated with isotype-matched antibodies were used as the negative control.

Statistical analysis. All data are presented as the mean \pm standard deviation. Levene's test was conducted for equal variances, one-way analysis of variance (ANOVA) for multi-group analysis, and the $t$-test for all other analyses. SPSS software, version 18.0 (SPSS, Inc., Chicago, IL, USA) was used to conduct the analysis. $\mathrm{P}<0.05$ was considered to indicate a statistically significant difference.

\section{Results}

Mouse model of SP meningitis. As evidenced by black ink staining, fluid injected at the base of the skull rapidly distributed throughout the cerebrospinal fluid (CSF; Fig. 1).

Assessment of the clinical disease status of mice with SP meningitis. Within 18 and $72 \mathrm{~h}$ post SP inoculation, all infected 

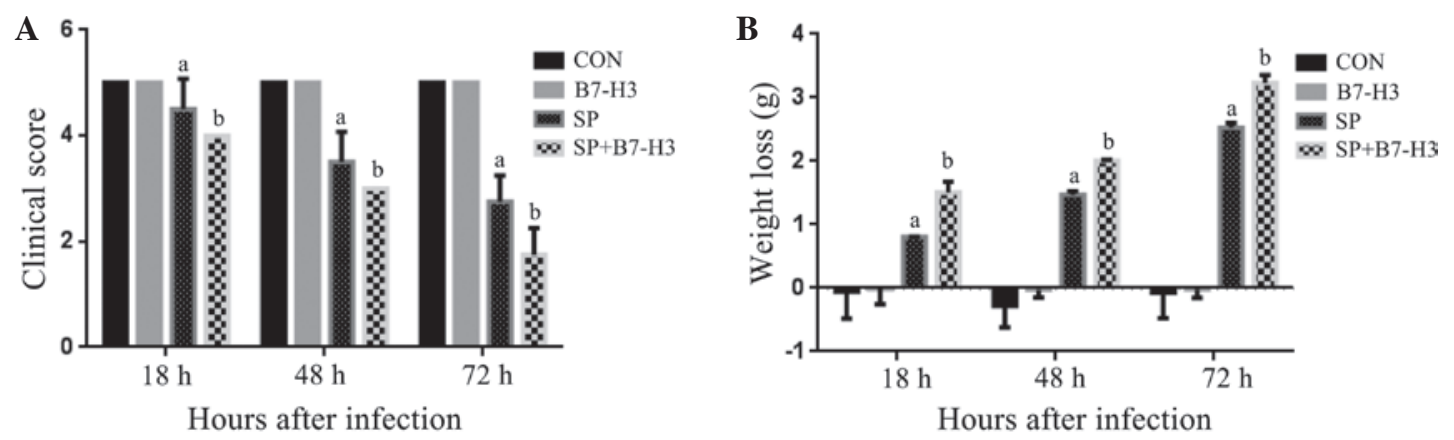

Figure 2. B7-H3 (A) deteriorates the clinical disease status and (B) causes body weight loss in Streptococcus pneumoniae-infected mice. BALB/c mice were challenged with NS as the control, B7-H3, live S. pneumoniae or live S. pneumoniae plus B7-H3 via intracerebroventricular injection. The clinical disease status and body weight loss were examined at 18,48 and $72 \mathrm{~h}$ post challenge as described in Materials and methods. Data are from 4-6 mice per time-point and represent 3-4 separate experiments. ${ }^{a} \mathrm{P}<0.05$ vs. mice that received NS, ${ }^{b} \mathrm{P}<0.05$ vs. mice that received S. pneumoniae alone. B7-H3, B7 homolog 3; NS, normal saline; CON, control; SP, Streptococcus pneumoniae.
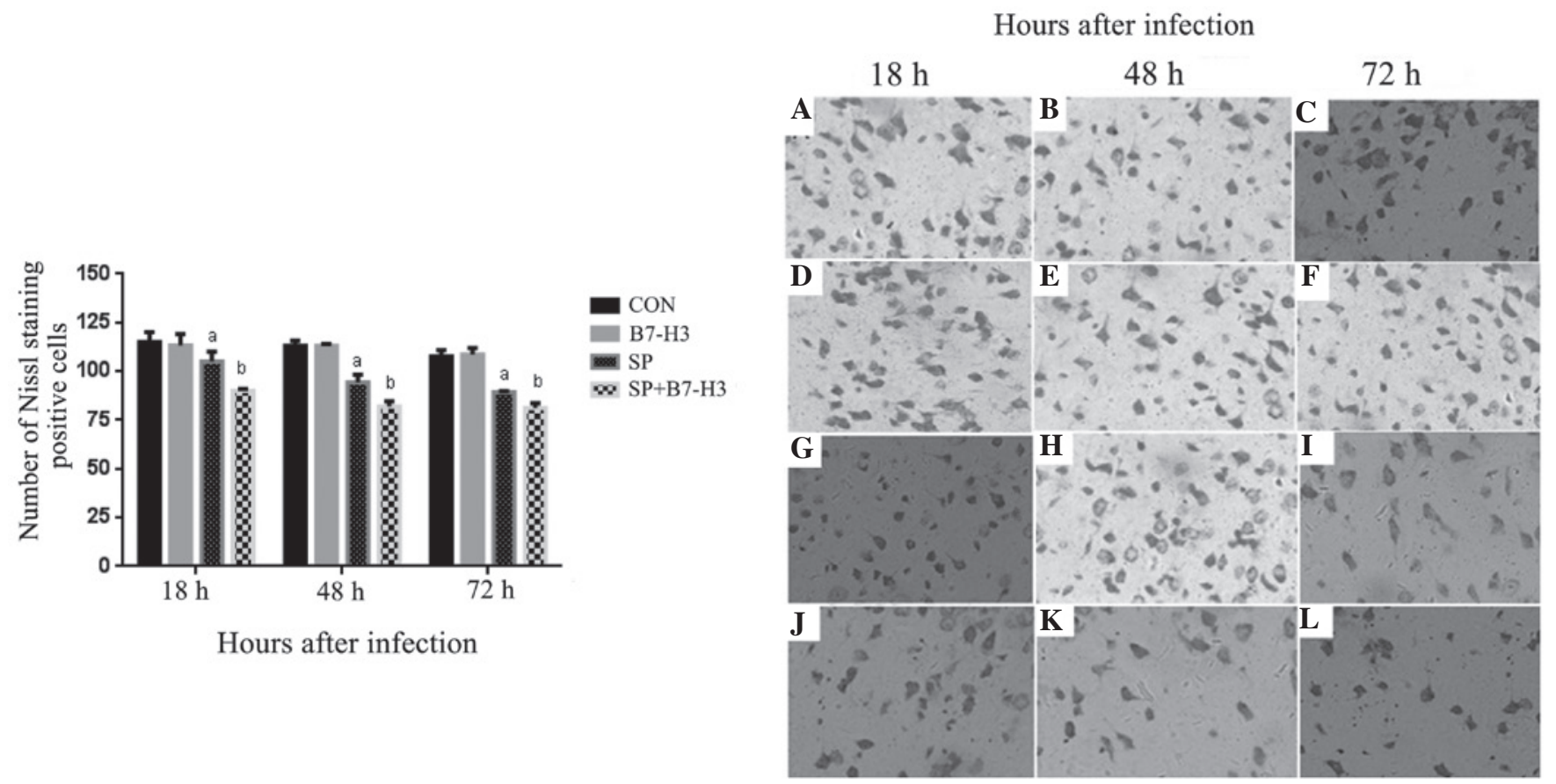

Figure 3. B7-H3 promotes neuronal loss and injury in brain tissues during Streptococcus pneumoniae-infected meningitis. BALB/c mice were challenged with NS as the control, B7-H3, live S. pneumoniae or live S. pneumoniae plus B7-H3 via intracerebroventricular injection. Brains were harvested from (A-C) NS-treated, (D-F) B7-H3-treated, (G-I) S. pneumoniae-infected and (J-L) S. pneumoniae plus B7-H3-infected mice, and brain sections were subjected to Nissl staining as described in Materials and methods. Representative immunohistochemical analyses are shown (magnification, x400). B7-H3 treatment led to significantly reduced Nissl staining of positive neurons or a greater number of injured neurons identified by the loss of Nissl substance, shrinkage with condensed nuclei and sparse Nissl bodies at (J) 18 (K) 48 and (L) 72 h after S. pneumoniae infection compared with that observed for S. pneumoniae alone (G-I, respectively). Data in the bar graph are expressed as mean \pm standard deviation of 6 mice per time-point and represent 3 separate experiments. ${ }^{a} \mathrm{P}<0.05 \mathrm{vs}$. mice that received NS, ${ }^{b} \mathrm{P}<0.05$ vs. mice that received S. pneumoniae alone. B7-H3, B7 homolog 3; NS, normal saline; CON, control; SP, Streptococcus pneumoniae.

mice displayed signs of disease represented by decreased spontaneous motor activity (Fig. 2A) and body weight loss (Fig. 2B) compared with NS-injected mice. Moreover, the combined treatment of mice with SP and B7-H3 further exaggerated the severity of the disease with significantly decreased spontaneous motor activity and increased body weight loss $(\mathrm{P}<0.05$ in comparison with that in the mice challenged with SP alone; Fig. 2). B7-H3 alone did not affect the clinical disease status (Fig. 2).

Nissl staining. To determine whether B7-H3 exacerbates the neuronal injury in SP-induced meningitis, brain tissue cytoarchitecture was evaluated using Nissl staining. The results revealed marked damage in terms of cell death in brain tissues between 18 and $72 \mathrm{~h}$ after SP infection. The majority of the damaged neurons exhibited shrinkage with condensed nuclei and sparse Nissl bodies (Fig. 3). Moreover, more damaged neurons were observed in the SP plus B7-H3 group compared with the SP group (Fig. 3). Quantitative data demonstrated that the numbers of Nisslpositive cells were significantly decreased in the SP group at 18,48 and $72 \mathrm{~h}$ post SP infection, and this effect was further augmented by $\mathrm{B} 7-\mathrm{H} 3$ administration $(\mathrm{P}<0.05$ vs. SP alone; Fig. 3). 


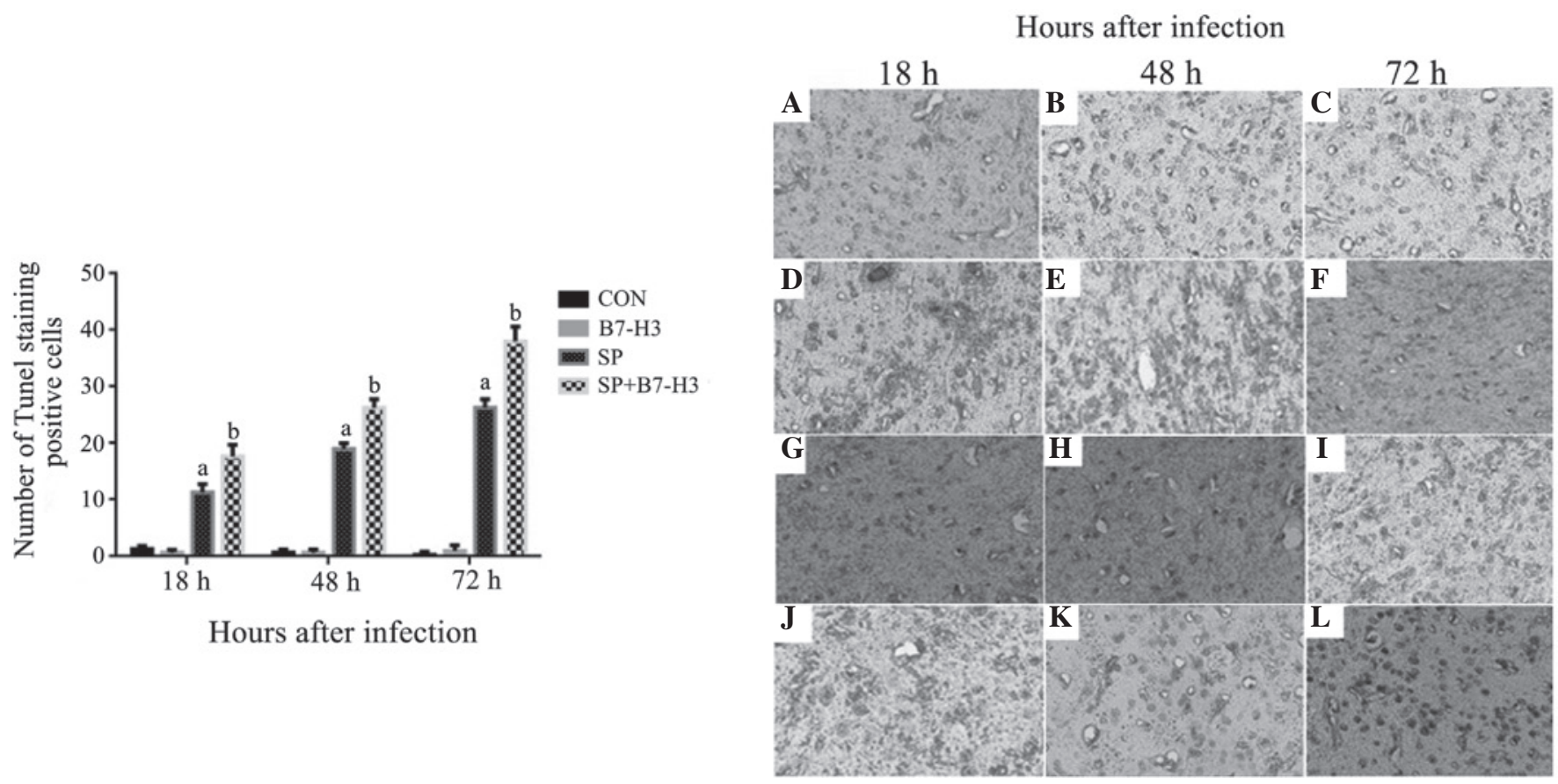

Figure 4. B7-H3 promotes neuronal loss and injury in brain tissues during Streptococcus pneumoniae-infected meningitis. BALB/c mice were challenged with NS as the control, B7-H3, live S. pneumoniae or live S. pneumoniae plus B7-H3 via intracerebroventricular injection. Brains were harvested from (A-C) NS-treated, (D-F) B7-H3-treated (G-I) S. pneumoniae-infected and (J-L) S. pneumoniae plus B7-H3-infected mice, and brain sections were subjected to TUNEL staining as described in materials and methods. Representative immunohistochemical analyses are shown (magnification, x200). S. pneumoniae plus B7-H3 treatment led to significantly increased apoptosis at (J) 18, (K) 48 and (L) $72 \mathrm{~h}$ after S. pneumoniae infection compared with S. pneumoniae infection alone (G-I, respectively). The data in the bar chart are expressed as mean \pm standard deviation of 6 mice per time-point and represent 3 separate experiments. ${ }^{\mathrm{a}} \mathrm{P}<0.05$ vs. mice that received NS, ${ }^{\mathrm{b}} \mathrm{P}<0.05$ vs. mice that received S. pneumoniae alone. B7-H3, B7 homolog 3; NS, normal saline; CON, control; SP, Streptococcus pneumoniae; TUNEL, terminal deoxynucleotidyl transferase-mediated dUTP nick end-labeling.

TUNEL staining. Apoptotic death was examined using TUNEL staining. The number of apoptotic cells in the brain tissues was significantly increased in the SP group at 18, 48 and $72 \mathrm{~h}$ after SP inoculation compared with that in the control group $(\mathrm{P}<0.05$; Fig. 4). Importantly, a substantially greater number of apoptotic cells were observed in the brain tissues of the mice challenged with SP plus B7-H3 than in the mice challenged with SP alone at all measured time-points $(\mathrm{P}<0.05$; Fig. 4), indicating that challenge with B7-H3 exacerbates SP-induced apoptotic cell death in the brain.

Immunohistochemical analysis of NSE and S100B expression. Having considered that NSE and S100B are biomarkers of brain injury in cerebral infections (9), protein expression of NSE and S100B was assessed in the brain tissues of SP-infected mice by immunochemical staining. While significantly decreased NSE-positive cells in brain tissues were observed at 18, 48 and $72 \mathrm{~h}$ after SP infection in the mice challenged with SP alone compared with the control group $(\mathrm{P}<0.05$; Fig. 5), mice receiving a combination of $\mathrm{SP}$ and B7-H3 displayed further significant reductions in NSE-positive cells at 18, 48 and $72 \mathrm{~h}$ after SP infection $(\mathrm{P}<0.05$ vs. mice challenged with SP alone; Fig. 5). SP infection led to increased protein expression of S100B at 18, 48 and $72 \mathrm{~h}$ after infection (Fig. 6). Notably, a substantial further accumulation of S100B protein in the brain was evident in mice challenged with a combination of SP and B7-H3 at all measured time-points $(\mathrm{P}<0.05$ vs. mice challenged with SP alone) (Fig. 6).

\section{Discussion}

SP infection resulted in significantly decreased expression of NSE at 18, 48 and $72 \mathrm{~h}$ post SP inoculation, and combined $\mathrm{SP}$ and B7-H3 challenge further reduced NSE expression levels when compared with that induced by SP infection alone. The expression of S100B was substantially increased in SP-infected mice, and was further elevated in the mice treated with SP and B7-H3. Furthermore, similar expression levels of NSE and S100B were observed in the B7-H3 group and the control group. These results indicate that $\mathrm{SP}$ infection results in neurological damage, and that the administration of B7-H3 does not cause neurological damage directly but aggravates SP infection-induced brain injury.

NSE ( $\gamma$-enolase) is a stable cell-specific isoenzyme of the glycolytic enzyme enolase, a dimer protein consisting of $\alpha$, $\beta$ and $\gamma$ subunits. In the brain, NSE is concentrated exclusively in the cytoplasm of neurons (10) and has been suggested to be a specific serum marker for neuronal damage. In the results of the present study, the reduction of NSE expression in the brain tissues of the mice treated with SP plus B7-H3 may reflect the following: i) NSE is primarily localized in the cytoplasm of neurons (10) and is not secreted; thus, an increase in its levels in the CSF or blood indicates structural damage of neuronal cells (8); ii) The number of existing functional neurons in the brain tissues that are available to be stained as NSE-positive by immunohistochemical analysis is markedly decreased due to apoptosis or necrosis caused by multiple factors exerting injurious effects, including exacerbated inflammation in 


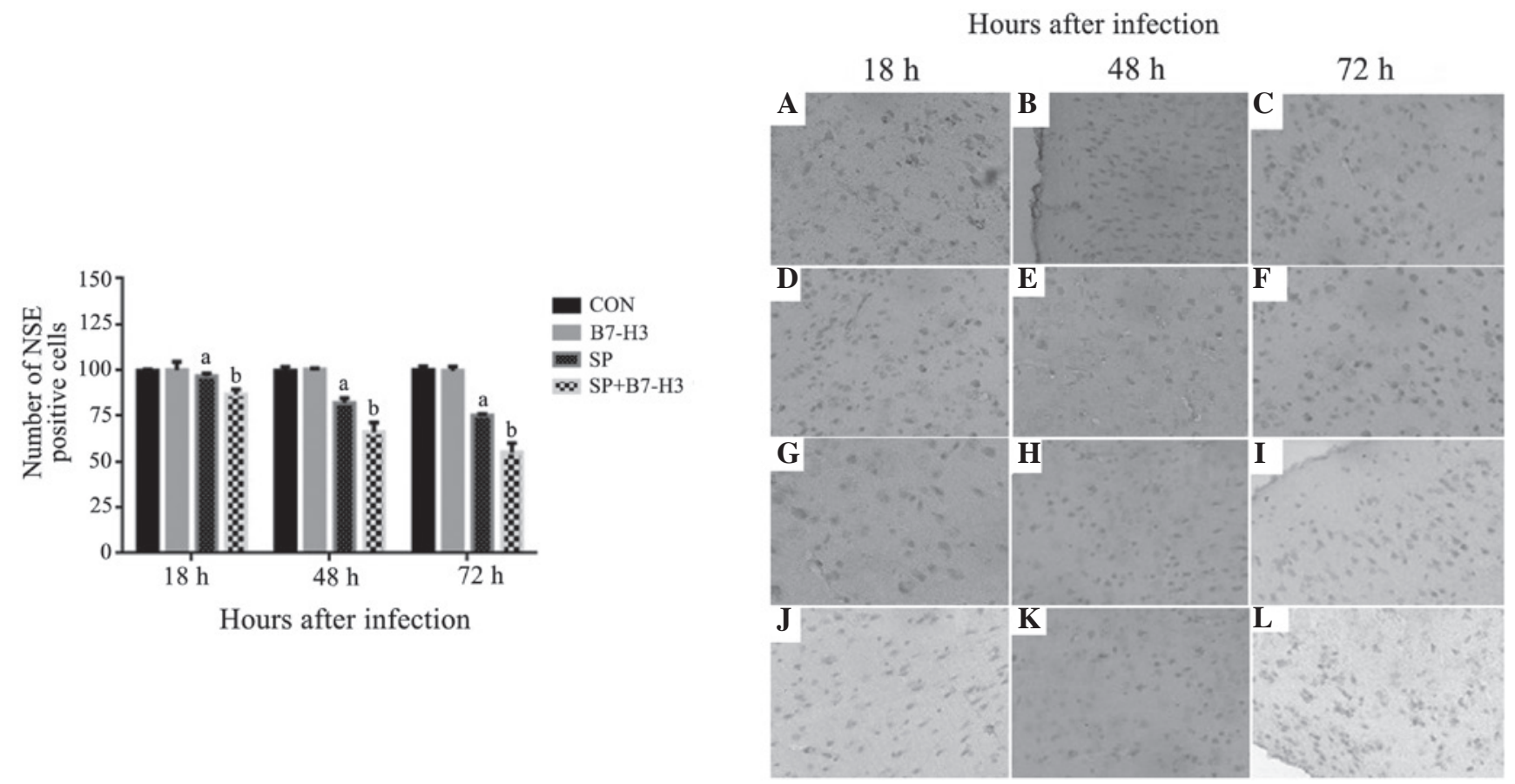

Figure 5. Efect of B7-H3 on the protein expression of NSE in the brain tissues of Streptococcus pneumoniae-infected mice. BALB/c mice were challenged with NS as the control, B7-H3, live S. pneumoniae or live S. pneumoniae plus B7-H3 via intracerebroventricular injection. Brains were harvested from the mice challenged with (A-C) NS, (D-F) B7-H3, (G-I) S. pneumoniae and (J-L) S. pneumoniae plus B7-H3 and the expression of NSE in the brain tissue was assessed by immunohistochemistry as described in materials and methods. Representative immunohistochemical analyses are shown (magnification, x200). The number of NSE-positive cells differed significantly between the S. pneumoniae plus B7-H3 group at (J) 18, (K) 48 and (L) $72 \mathrm{~h}$ and the S. pneumoniae group at these time-points (G-I, respectively); there was no significant difference in the number of S100B-positive cells between the control group at (A) 18, (B) 48 and (C) $72 \mathrm{~h}$ and the B7-H3 group at these time-points (D-F, respectively). The data in the bar graph are expressed as the mean \pm standard deviation of 6 mice per time-point and represent 3 separate experiments. ${ }^{\mathrm{a}} \mathrm{P}<0.05$ vs. mice that received NS, ${ }^{\mathrm{b}} \mathrm{P}<0.05$ vs. mice that received S. pneumoniae alone. B7-H3, B7 homolog 3; NSE, neuron-specific enolase; NS, normal saline; CON, control; SP, Streptococcus pneumoniae.

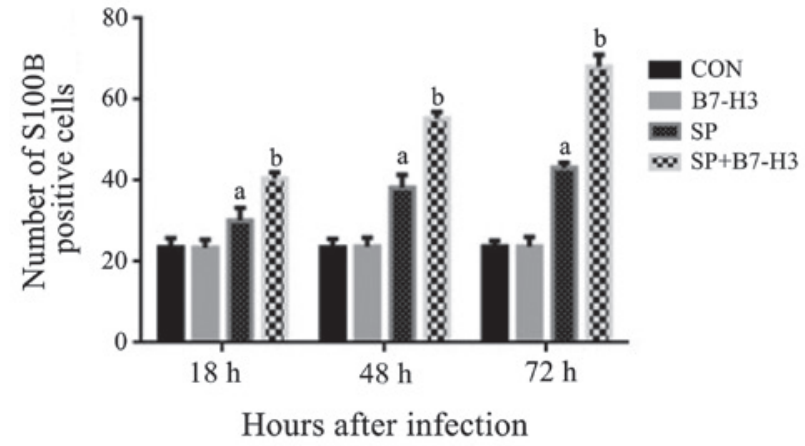

Hours after infection

\section{Hours after infection}

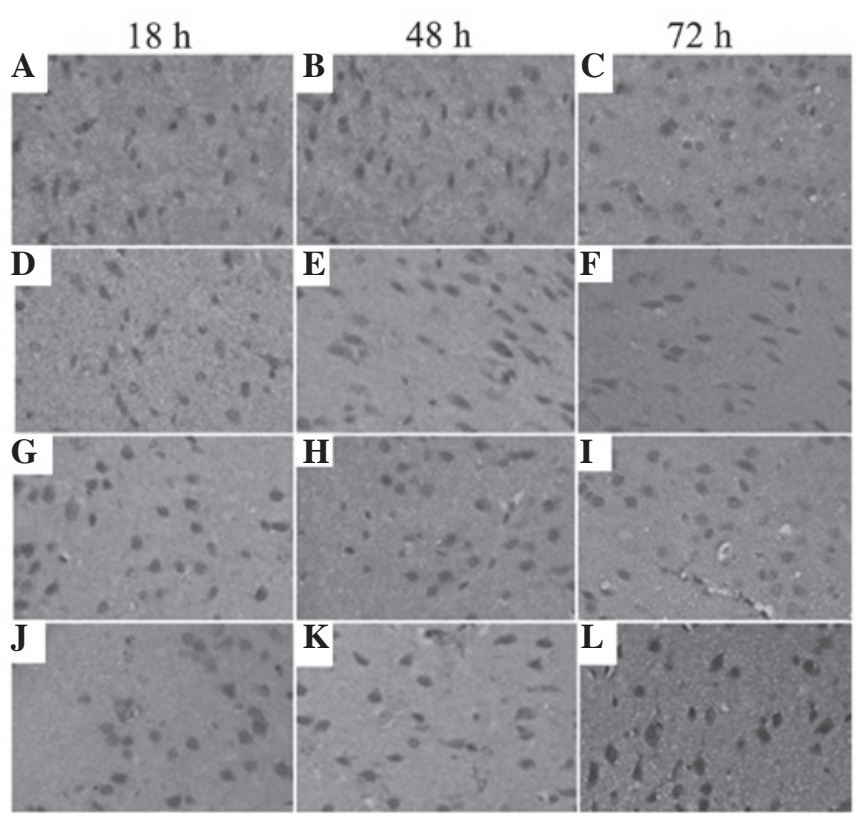

Figure 6. B7-H3 upregulates S100B expression in the cerebral tissues of Streptococcus pneumoniae-infected mice. BALB/c mice were challenged with NS as the control, B7-H3, live S. pneumoniae or live S. pneumoniae plus B7-H3 via intracerebroventricular injection. Brains were harvested from (A-C) NS, (D-F) B7-H3, (G-I) S. pneumoniae and (J-L) S. pneumoniae plus B7-H3 challenged mice and the expression of S100B in the brain was assessed by immunohistochemistry as described in materials and methods. Representative immunohistochemical analyses are shown (magnification, $\mathrm{x} 400$ ). The number of S100B-positive cells differed significantly between the S. pneumoniae plus B7-H3 group at (J) 18, (K) 48 (L) and $72 \mathrm{~h}$, and the S. pneumoniae group at these time-points (G-I, respectively); there was no significant difference in the number of S100B-positive cells between the control group at (A) 18 , (B) 48 and (C) $72 \mathrm{~h}$ and the B7-H3 group at these time-points (D-F, respectively) $72 \mathrm{~h}$. The data in the bar chart are expressed as the mean \pm standard deviation of 6 mice per time-point and represent 3 separate experiments. ${ }^{a} \mathrm{P}<0.05$ vs. mice that received NS, ${ }^{b} \mathrm{P}<0.05$ vs. mice that received S. pneumoniae alone. B7-H3, B7 homolog 3; NS, normal saline; CON, control; SP, Streptococcus pneumoniae. 
response to bacteria stimuli (5), oxidative stress, lipid peroxidation, mitochondrial damage and BBB breakdown (1). Further reductions in NSE expression in brain tissues in response to combined SP and B7-H3 challenge indicate a greater degree of neuronal loss and structural neuronal damage, which is consistent with our previous study where it was identified that B7-H3 exacerbated the SP infection-induced proinflammatory response and BBB disruption (6).

S100B belongs to the multi-genic family of $\mathrm{Ca}^{2+}$-binding proteins and is abundant in astrocytes, where it is found diffusely in the cytoplasm and is associated with membranes and cytoskeleton constituents (12). Extensive evidence indicates that S100B in high levels (micromolar) may contribute to the severity of and neurological damage during bacterial meningitis $(13,14)$. As a biomarker that indicates damage or dysfunction of the CNS (15), S100B levels could be used as an additional monitoring parameter in CNS infection (16). In the present study, the significantly higher level of expression of S100B in brain tissues in the B7-H3 plus SP group compared with that in the SP group indicates that B7-H3 aggravates SP infection-induced neurological damage, since elevated S100B expression reflects an ongoing cellular injury (specifically of astrocytes) (17,18). Furthermore, persistent increases in S100B may have a neurotoxic effect by inducing apoptosis, causing the release of proinflammatory cytokines as well as nitric oxide from astroglial cells, and exhibiting detrimental effects on neurons $(13,19)$. Consistent with the dynamic changes of S100B expression, a greater amount of neuronal apoptosis and/or loss in the brain tissues, and a worse clinical status were observed from 18 to $72 \mathrm{~h}$ after SP infection. Administration of B7-H3 exacerbated SP infection-induced apoptosis or loss of neurons and clinical status, demonstrating that B7-H3 plays an important role in the pathological process of SP-induced brain injury.

A number of studies have demonstrated that neurons in the CNS undergo apoptotic cell death during SP-induced bacterial meningitis (20-23). Consistent with this, the B7-H3-amplified cell apoptosis and neuronal loss as demonstrated by TUNEL and Nissl staining in the present study are also associated with deteriorated clinical disease status. Challenge with B7-H3 caused a further increase in apoptotic cell death and increased the severity of disease with significantly impaired spontaneous motor activity and increased body weight loss in SP-infected mice. Thus, these in vivo data indicate that B7-H3 not only amplifies the SP infection-initiated expression of neuronal (NSE) and glial (S100B) markers, but also results in aggravated cell apoptosis in the CNS, suggesting that B7-H3 plays a detrimental role in the development of SP meningitis and its intracranial complications.

SP is recognized by antigen-presenting cells binding to Toll-like receptor 2 (TLR2), one of the main pattern recognition receptors that lead to the activation of $\mathrm{NF}-\kappa \mathrm{B}(6,24)$ contributing to the transcription of numerous genes involved in the pathogenesis of SP meningitis, such as tumor necrosis factor- $\alpha$, interleukin-1 $\beta$ (6), inducible nitric oxide synthase, and intercellular adhesion molecules $(25,26)$. Our previous study (6) demonstrated that B7-H3 participates in the development of SP infection-induced bacterial meningitis by amplifying the inflammatory response and exacerbating $\mathrm{BBB}$ disruption through a TLR2-dependent mechanism. Consistent with the above findings, the present study demonstrates that the administration of B7-H3 leads to increased cell apoptosis, upregulated S100B expression and downregulated NSE expression in brain tissues, which are probably associated with the ongoing loss of neurons.

In summary, the present study documents a critical role of B7-H3 in the pathogenesis of neuronal injury in a model of SP meningitis. As part of a series of studies on the effects of B7-H3 on the development of SP meningitis, these results may contribute to further understanding of the factors involved in the pathogenesis of brain in bacterial meningitis, and may have further clinical implications. Other adjunctive approaches targeting B7-H3 may be useful for altering the outcome of SP-induced meningitis.

\section{Acknowledgements}

The authors would like to thank Xueming Zhu, Yunzhen Tao and Bin Zhou for the excellent technical support and Yumin $\mathrm{Hu}$ for mice breeding and care. This study was supported by grants from the National Natural Science Foundation of China (no. 81273242, 81272143 and 81301494), Natural Science Foundation of Jiangsu Province (no. BK2012605), Jiangsu Province Program of Innovative and Entrepreneurial Talents (2011-2014) and Priority Academic Program Development of Jiangsu Higher Education Institutions.

\section{References}

1. Barichello T, Generoso JS, Collodel A, Moreira AP and Almeida SM: Pathophysiology of acute meningitis caused by Streptococcus pneumoniae and adjunctive therapy approaches. Arq Neuropsiquiatr 70: 366-372, 2012.

2. Shapiro MA, Donovan KD and Gage JW: Comparative therapeutic efficacy of clinafloxacin in a pneumococcal meningitis mouse model. J Antimicrob Chemother 45: 489-492, 2000.

3. Streitbürger DP, Arelin K, Kratzsch J, Thiery J, Steiner J, Villringer A, Mueller K and Schroeter ML: Validating serum S100B and neuron-specific enolase as biomarkers for the human brain - A combined serum, gene expression and MRI study. PLoS One. 7: e43284, 2012

4. Zhao X, Li DC, Zhu XG, et al: B7-H3 overexpression in pancreatic cancer promotes tumor progression. Int J Mol Med 31: 283-91, 2013.

5. Chen X, Zhang G, Li Y, Feng X, Wan F, Zhang L, Wang J and Zhang X: Circulating B7-H3(CD276) elevations in cerebrospinal fluid and plasma of children with bacterial meningitis. J Mol Neurosci 37: 86-94, 2009.

6. Chen X, Quinn EM, Ni H, Wang J, Blankson S, Redmond HP, Wang JH and Feng X: B7-H3 participates in the development of experimental pneumococcal meningitis by augmentation of the inflammatory response via a TLR2-dependent mechanism. J Immunol 189: 347-355, 2012.

7. Leib SL, Clements JM, Lindberg RL, et al: Inhibition of matrix metalloproteinases and tumour necrosis factor alpha converting enzyme as adjuvant therapy in pneumococcal meningitis. Brain 124: 1734-1742, 2001

8. Wang Q, Wang C, Shu Z, et al: Valeriana amurensis improves Amyloid-beta 1-42 induced cognitive deficit by enhancing cerebral holinergic function and protecting the brain neurons from apoptosis in mice. J Ethnopharmacol 153: 318-325, 2014.

9. Rohlwink UK and Figaji AA: Biomarkers of brain injury in cerebral infections. Clin Chem 60: 823-834, 2014.

10. Marangos PJ and Schmechel DE: Neuron specific enolase, a clinically useful marker for neurons and neuroendocrine cells. Annu Rev Neurosci 10: 269-295, 1987.

11. El-Naga RN: Ahmed HI2 and Abd Al Haleem EN: Effects of indole-3-carbinol on clonidine-induced neurotoxicity in rats: Impact on oxidative stress, inflammation, apoptosis and monoamine levels. Neurotoxicology 44: 48-57, 2014. 
12. Adami C, Sorci G, Blasi E, Agneletti AL, Bistoni F and Donato R S100B expression in and effects on microglia. Glia 33: 131-142, 2001.

13. Hamed SA, Hamed EA and Zakary MM: Oxidative stress and $\mathrm{S}-100 \mathrm{~B}$ protein in children with bacterial meningitis. BMC Neurol 9: 51, 2009.

14. Park JW, Suh GI and Shin HE: Association between cerebrospinal fluid S100B protein and neuronal damage in patients with central nervous system infections. Yonsei Med J 54: 567-71, 2013

15. Yang XY, Lin J, Lu XY, Zhao XY: Expression of S100B protein levels in serum and cerebrospinal fluid with different forms of neuropsychiatric systemic lupus erythematosus. Clin Rheumatol 27: 353-357, 2008.

16. Lins H, Wallesch CW and Wunderlich MT: Sequential analyses of neurobiochemical markers of cerebral damage in cerebrospinal fluid and serum in CNS infections. Acta Neurol Scand 112: 303-308, 2005

17. Raabe A and Seifert V: Protein S-100B as a serum marker of brain damage in severe head injury: Preliminary results. Neurosurg Rev 23: 136-138, 2000.

18. Raabe A and Seifert V: Fatal secondary increase in serum S-100B protein after severe head injury. Report of three cases. J Neurosurg 91: 875-877, 1999.

19. Steiner J, Bernstein HG, Bielau H, Berndt A, Brisch R, Mawrin $\mathrm{C}$ et al: Evidence for a wide extraastrocytic distribution of S100B in human brain. BMC Neurosci 8: 2, 2007.
20. Leib SL, Kim YS, Chow LL, Sheldon RA and Täuber MG: Reactive oxygen intermediates contribute to necrotic and apoptotic neuronal injury in an infant rat model of bacterial meningitis due to group B streptococci. J Clin Invest 98: 2632-2639, 1996.

21. Tauber SC, Harms K, Falkenburger B, Weis J, Sellhaus B, Nau R, Schulz JB and Reich A: Modulation of hippocampal neuroplasticity by Fas/CD95 regulatory protein 2 (Faim2) in the course of bacterial meningitis. J Neuropathol Exp Neurol 73: 2-13, 2014.

22. Zysset-Burri DC, Bellac CL, Leib SL and Wittwer M: Vitamin B6 reduces hippocampal apoptosis in experimental pneumococcal meningitis. BMC Infect Dis 13: 393, 2013.

23. Braun JS, Sublett JE, Freyer D, Mitchell TJ, Cleveland JL, Tuomanen EI and Weber JR: Pneumococcal pneumolysin and $\mathrm{H}_{2} \mathrm{O}_{2}$ mediate brain cell apoptosis during meningitis. J Clin Invest 109: 19-27, 2002.

24. Opitz B, Eitel J, Meixenberger K and Suttorp N: Role of Toll-like receptors, NOD-like receptors and RIG-I-like receptors in endothelial cells and systemic infections. Thromb Haemost 102: 1103-1109, 2009.

25. Koedel U, Bayerlei I, Paul R, Sporer B and Pfister HW: Pharmacologic interference with NF-kappaB activation attenuates central nervous system complications in experimental pneumococcal meningitis. J Infect Dis 182: 1437-1445, 2000.

26. Kastenbauer S, Koedel U, Weih F, Ziegler-Heitbrock L and Pfister HW: Protective role of NF-kappaB1 (p50) in experimental pneumococcal meningitis. Eur J Pharmacol 498: 315-318, 2004. 